\title{
Local- and Intermediate-Range Structures on Ordinary and Exotic Phase-Change Materials by Anomalous X-ray Scattering
}

\author{
Jens Rüdiger STEllhorn, ${ }^{* 1}$ Shinya HosoKawa, ${ }^{* 2 \dagger}$ and Shinji KoHARA $* 3, * 4, * 5$ \\ *1 Department of Applied Chemistry, Hiroshima University, 1-4-1 Kagamiyama, Higashi-Hiroshima 739-8527, \\ Japan \\ *2 Department of Physics, Kumamoto University, 2-39-1 Kurokami, Chuo, Kumamoto 860-8555, Japan \\ *3 Research Center for Advanced Measurement and Characterization, National Institute for Materials Science \\ (NIMS), 1-1-1 Kouto, Sayo, Hyogo 679-5148, Japan \\ *4 Center for Materials Research by Information Integration $\left(C M I^{2}\right)$, Research and Services Division of Materials \\ Data and Integrated System (MaDIS), National Institute for Materials Science (NIMS), 1-2-1 Sengen, \\ Tsukuba, Ibaraki 305-0047, Japan \\ *5 PRESTO, Japan Science and Technology Agency, 7 Gobancho, Chiyoda, Tokyo 102-0076, Japan
}

\begin{abstract}
Local- and intermediate-range atomic structures were investigated on amorphous phases of an ordinary phase-change material, $\mathrm{Ge}_{2} \mathrm{Sb}_{2} \mathrm{Te}_{5}$ (GST), and an exotic one, $\mathrm{Cu}_{2} \mathrm{GeTe}_{3}$ (CGT), by using anomalous X-ray scattering close to $K$ absorption edges of each element to find a fast amorphous-crystalline phase-change mechanism. The obtained data were analyzed by using reverse Monte Carlo modeling to obtain partial structure factors, partial pair distribution functions, and threedimensional atomic configurations. Ring statistics were carefully examined to clarify the similarity and difference compared with the corresponding crystal structures, and it was found that amorphous GST has a number of fourmembered rings indicating fragments of crystal structure, and amorphous CGT has a remarkable number of threemembered rings showing a collapse of crystal structures composed of purely six-membered rings. A persistent homology analysis was carried out and long-range ring structures of the constituent elements were observed in the amorphous phase, which may originate from fragments of crystal structures with a long-range periodicity.
\end{abstract}

Keywords Amorphous, phase change materials, atomic structure, intermediate-range, reverse Monte Carlo analysis, homology analysis

(Received September 14, 2019; Accepted December 10, 2019; Advance Publication Released Online by J-STAGE December 20, 2019)
1 Introduction
2 Experimental Procedure
3 Data Analysis
3.1 Anomalous X-ray scattering
3.2 Reverse Monte Carlo modeling
3.3 Persistent homology analysis

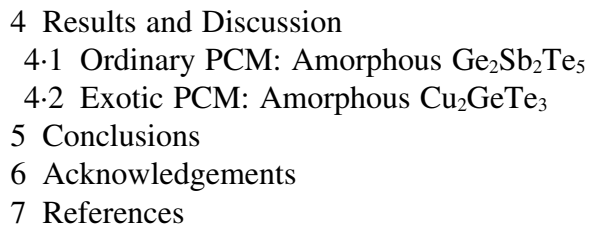

7

\section{Jens Rüdiger StellhorN received his Ph.D. degree in 2015 from the Philipps Univer- sity of Marburg (Germany). He worked as a postdoctoral researcher in Nagoya Institute of Technology (2016), in Kumamoto University (2016 -} 2018), at the German Electron Synchrotron DESY Marburg (1995 - 2004), Hiroshima Institute of (2018-2019) and as Assistant Professor in Technology (2004-2012), and Kumamoto University Hiroshima University (2019-). His research interests $(2012-)$. His research topics are structure and cover the investigations of local structures on dynamics of non-crystalline materials, and atomic scales in a variety of systems, in particular recently randomness in crystals by synchrotron regarding amorphous and crystalline states in radiation experiments. functional materials.

† To whom correspondence should be addressed.

E-mail: shhosokawa@kumamoto-u.ac.jp
Shinya HosoKawa is a Professor of the Department of Physics, Kumamoto University. He received his Doctor of Science degree in 1986 from Kyoto University. He worked at Hiroshima University

\section{.} he worked as a postdoctoral fellow at Japan Synchrotron Radiation Research Institute (JASRI),
where he was involved in the development of dedicated hard X-ray diffractometer for glass, liquid, and amorphous materials at SPring-8. From 2001 to 2015, he worked as a beamline scientist at SPring-8. Since 2015, he has been working at NIMS. His current research interests include the inorganic chemistry and materials science of glassy, liquid, and amorphous materials. 
(a)

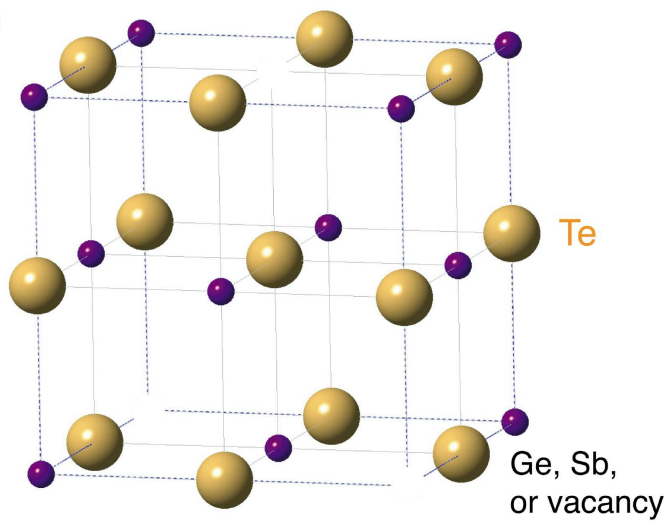

(b)

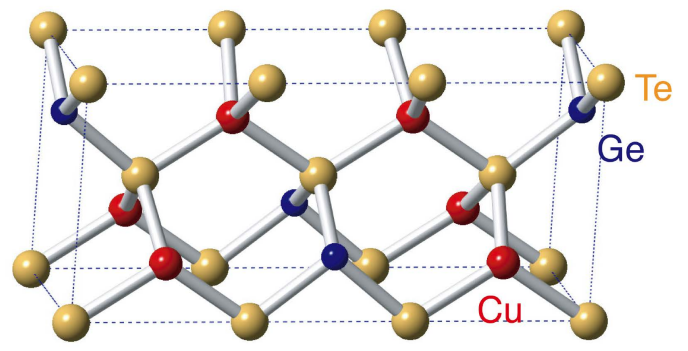

Fig. 1 Atomic structures of crystalline (a) GST ${ }^{4}$ and (b) CGT $^{17}$ after Ref. 24.

\section{Introduction}

Rewritable optical storage devices, like digital versatile diskrandom access memory (DVD-RAM) or blu-ray, have become common media for data storage and are widely used in all areas of daily life, such as video recording. The writing/erasing process on these devices is attained by a reversible laser-induced crystalline-amorphous phase transition of the so-called phase change materials (PCM). The transition occurs very fast, i.e., on a time scale of a few nanoseconds, and is accompanied by a significant change in the optical and electrical properties. On the other hand, both of the phases must be sufficiently stable for more than 10 years at ambient conditions. These properties are an excellent basis for the reversible data storage ability.

$\mathrm{Ge}-\mathrm{Sb}-\mathrm{Te}$ alloys are at present raw materials used widely for commercial optical storage disks, ${ }^{1}$ in which $\mathrm{Ge}_{2} \mathrm{Sb}_{2} \mathrm{Te}_{5}$ (GST) is a typical alloy. In the crystalline phase, these alloys have a larger optical reflectance ${ }^{2}$ and a higher electrical conductivity ${ }^{3}$ compared with those in the corresponding amorphous phase. From these properties, we refer to this alloy as an ordinary PCM.

The atomic structure of crystalline GST film can be wellunderstood by an X-ray diffraction (XRD) experiment. ${ }^{4}$ It does not exhibit the stable crystal structure of hexagonal form in ambient conditions, but a metastable rock salt structure, with Te atoms occupying sites on one face-centered-cubic $(f c c)$ sublattice and with $\mathrm{Ge}, \mathrm{Sb}$, and $20 \%$ of vacancies forming another $f c c$ sublattice as shown in Fig. 1(a). Convincing evidence for pronounced lattice distortions has been found in an X-ray absorption fine structure (XAFS) experiment by Kolobov et al., ${ }^{5}$ where six Ge-Te neighboring bonds of octahedral symmetry sites separate into three short and three long bonds as in GeTe crystal. $^{6}$

An atomic structure of amorphous GST was also explored with XAFS by Kolobov et al., ${ }^{5}$ who found remarkable decreases of $\mathrm{Ge}-\mathrm{Te}$ and $\mathrm{Sb}-\mathrm{Te}$ covalent bond lengths from those in the crystal. The XAFS data also indicated a change in a coordination number around Ge from sixfold in the crystal to fourfold in the amorphous. This observation in combination with the abovementioned lattice distortions in the crystal, led the authors to propose an umbrella flip model for the fast transition of the GST, where the Ge atoms flip from an octahedral arrangement in the crystal to a tetrahedral environment in the amorphous phase. Another XAFS study ${ }^{7}$ gave a different local structure around the Ge atoms, suggesting that a significant fraction of the Ge-Ge wrong bonds exists in addition to usual Ge-Te bonds. Furthermore, an environment around the $\mathrm{Sb}$ atoms was discussed to be mainly threefold coordinated by $\mathrm{Te}$ atoms, like the distorted rock salt crystal.

Kohara et al. ${ }^{8}$ measured the total structure factor, $S(Q)$, of amorphous GST using high-energy XRD, and analyzed the data using reverse Monte Carlo (RMC) modeling. They concluded that the resemblance of an even-membered ring structure is related to the fast phase change process. Their RMC calculation, however, had strong constraints, such as exclusions of cationcation and Te-Te wrong bonds, because the experimental input was only a total $S(Q)$ spectrum. Therefore, a careful inspection is necessary for their above conclusion. By combining X-ray and neutron total scattering results with XAFS data, Jóvári et al. ${ }^{9}$ carried out an RMC analysis, with which the reliability of the RMC output may be improved. The XAFS data, however, do not help for investigating intermediate-range structures of amorphous GST because information on amorphous structures from XAFS is mostly limited to nearest neighboring atoms.

A molecular dynamics (MD) simulation with density functional theory (DFT) was performed by Akola and Jones, ${ }^{10}$ which shows a high degree of alternating four-membered rings ( $A B A B$ square rings) being the main building blocks for the metastable rock salt GST crystal. An $a b$ initio MD simulation was carried out by Hegedüs and Elliott, ${ }^{11}$ and they also found very high densities of square rings. These fragments of the crystal were considered to originate from the fast phase-change process. Although it has not yet been explicitly stated in these papers, it is often implied that the umbrella flip model is unlikely to be correct. On the other hand, the similarities of the local structures in the amorphous and crystalline phases intuitively indicate the lower stability of the phases, contradicting the actual property of the long-lasting DVD media.

Amorphous GST crystallizes at about $150^{\circ} \mathrm{C}$ into a cubic state, and then at about $350^{\circ} \mathrm{C}$ into a more stable hexagonal state..$^{3,12}$ It is well-known that GST needs a high reset (crystal to amorphous) current owing to its high melting point of about $630^{\circ} \mathrm{C},{ }^{13}$ requiring high power consumption. Moreover, the thermal stability of amorphous GST is not enough owing to the relatively low crystallization temperature of $150^{\circ} \mathrm{C}$, which is not satisfactory for high temperature applications, e.g., in automobiles.

Sutou et al. discovered a new PCM of $\mathrm{Cu}_{2} \mathrm{GeTe}_{3}$ (CGT), having a high crystallization temperature of about $250^{\circ} \mathrm{C}^{13}$ and a low melting point of about $500^{\circ} \mathrm{C},{ }^{14}$ which are suitable for hightemperature applications and low power consumption, respectively. Interestingly, upon crystallization, the optical reflectance decrease ${ }^{15}$ in contrast to GST, although the electrical resistivity decreases by an order of 2 as usual. ${ }^{16}$ Moreover, the density decreases upon crystallization. ${ }^{16}$ From these unusual properties on the phase change, we refer to this alloy as an exotic PCM.

An atomic structure of crystalline CGT consists of slightly distorted corner-sharing $\mathrm{CuTe}_{4}$ and $\mathrm{GeTe}_{4}$ tetrahedra with a 
space group Imm $2,{ }^{17}$ as shown in Fig. 1(b). As regards the ring statistics, crystalline CGT is composed of purely six-membered rings as shown in the figure.

Concerning the atomic structure of amorphous CGT, Jóvári et al. ${ }^{18}$ carried out XRD experiments and XAFS measurements close to the $\mathrm{Cu}, \mathrm{Ge}$, and $\mathrm{Te} K$ absorption edges, and the results were analyzed using RMC modeling. They found that there are significant numbers of the $\mathrm{Cu}-\mathrm{Cu}$ and $\mathrm{Ge}-\mathrm{Ge}$ wrong bonds, and the averaged partial coordination number around each element $i$, $\left\langle N_{\mathrm{i}}\right\rangle$, is close to four. They concluded that such high average coordination numbers of the network contribute to the enhanced thermal stability of amorphous CGT. Since these values of four are the same as those in crystalline CGT, however, the structural reason for their conclusion is doubtful.

A detailed XAFS investigation on the local structures of crystalline and amorphous CGT was carried out by Kamimura et al. ${ }^{19,20}$ and they found several curious features in the structures of CGT. In the crystal phase, the average $\mathrm{Cu}-\mathrm{Te}$ bond length is shorter than that of Ge-Te, opposite to the previous XRD results. ${ }^{17}$ As regards $\left\langle N_{\mathrm{i}}\right\rangle$ in the amorphous phase, values of $\sim 4.3$ and $\sim 4.8$ were obtained around the $\mathrm{Cu}$ and $\mathrm{Ge}$ atoms, larger than the crystal values of four. Moreover, X-ray absorption near edge structure (XANES) data near the $\mathrm{Cu} K$ edge indicate a large smearing-out on the amorphization in contrast to a similarity in the Ge $K$ edge XANES data, indicating large differences in the atomic configurations between two phases.

An $a b$ initio MD simulation was carried out on CGT by Skelton et al. ${ }^{21}$ to investigate the atomic-level structures and physical properties of both the phases. For amorphous CGT, three curious features were found, i.e., 1) sharp peaks in bond angle distributions, in particular around the $\mathrm{Cu}$ atoms, 2) very short average $\mathrm{Cu}-\mathrm{Te}$ interatomic distance of $0.263 \mathrm{~nm}$, which was much shorter than Ge-Te length of $0.280 \mathrm{~nm}$, and 3) dense $\mathrm{Cu}$-rich regions, which are different from the above XRD/ XAFS/RMC result. ${ }^{18}$ These features may be concluded to account for the experimentally observed increase in density on the amorphization and the exotic optical reflectance behavior. Another ab initio MD simulation was carried out by Chen et al., ${ }^{22}$ who tried to investigate the bonding chemistry in amorphous CGT. They found that there are large amounts of homopolar wrong bonds, and triangular clusters composed of threefold connections of the $\mathrm{Cu}$ atoms. The relationship between the $\mathrm{Cu} d$ and Te lone-pair electrons may be closely related to the exotic properties of CGT.

As mentioned above, the key experimental information on the intermediate-range atomic structures is still lacking in amorphous GST and CGT until our anomalous X-ray scattering (AXS) experiments. ${ }^{23,24}$ AXS experiments were carried out at incident X-ray energies close to the $\mathrm{Ge}, \mathrm{Sb}, \mathrm{Te}$, and $\mathrm{Cu} K$ absorption edges. With three sets of differential structure factors, $\Delta_{\mathrm{k}} S(Q)$, together with total $S(Q)$, RMC analyses were performed to obtain partial structure factors, $S_{\mathrm{ij}}(Q)$, partial pair distribution functions, $g_{\mathrm{ij}}(r)$, and three-dimensional atomic configurations. In this article, we would like to clarify the features of the fast phase-change natures of GST and CGT by obtaining detailed information about local- and intermediaterange atomic structures.

\section{Experimental Procedure}

Amorphous GST sample films were prepared by radio-frequency sputtering deposition from GeTe and $\mathrm{Sb}_{2} \mathrm{Te}_{3}$ targets on polycarbonate substrates. In the same way, amorphous CGT were manufactured from $\mathrm{CuTe}$ and $\mathrm{GeTe}$ targets on $\mathrm{SiO}_{2}$
$(20 \mathrm{~nm}) / \mathrm{Si}(0.7 \mathrm{~mm})$ substrates. ${ }^{25,26}$ The samples were scratched from the substrates, and contained between two thin-walled $(\sim 50 \mathrm{~m})$ sapphire plates or thin $(\sim 10 \mathrm{~m})$ Kapton foils with the sample thickness of $\sim 50 \mathrm{~m}$ for the AXS experiments near Ge and $\mathrm{Cu} K$ absorption edges and $\sim 0.2 \mathrm{~mm}$ for those near the $\mathrm{Sb}$ and Te $K$ edges.

AXS experiments were carried out by using a standard $\omega-2 \theta$ diffractometer installed at the beamline BM02 of the European Synchrotron Radiation Facility (ESRF) in Grenoble, France. The scattering experiments were performed at two incident $\mathrm{X}$-ray energies below each $K$ edge $(-20 \mathrm{eV}$ for $\mathrm{Ge}$ and $\mathrm{Cu}$, $-30 \mathrm{eV}$ for $\mathrm{Sb}$ and $\mathrm{Te}$, and $-200 \mathrm{eV}$ for all elements). For discriminating the elastic signal from the $K$ fluorescent and Compton scattering contributions, a bent graphite crystal analyzer was mounted on a 1-m long detector arm. The feasibility of this detecting system is described elsewhere..$^{27,28}$

\section{Data Analysis}

\section{3·1 Anomalous X-ray scattering}

AXS utilizes anomalous variations of atomic form factors, $f$, of a specific element near an X-ray absorption edge of the respective element. ${ }^{29}$ The complex $f$ is given as

$$
f(Q, E)=f_{0}(Q)+f^{\prime}(E)+i f^{\prime \prime}(E)
$$

where $f_{0}$ is the usual energy-independent term, and $f^{\prime}$ and $f^{\prime \prime}$ are real and imaginary parts of the anomalous term, respectively. When the incident X-ray energy approaches an absorption edge of a constituent element, $f^{\prime}$ has a large negative minimum and $f^{\prime \prime}$ shows an abrupt jump.

One can use a difference between two scattering spectra near an absorption edge of the $k$-th element, $\Delta_{\mathrm{k}} I$, where one is typically measured at an incident X-ray energy of some $10 \mathrm{eV}$ and one at some $100 \mathrm{eV}$ below the absorption edge ( $E_{\text {near }}$ and $E_{\text {far }}$, respectively). This difference is expressed as

$$
\alpha_{\mathrm{k}} \Delta_{\mathrm{k}} I\left(Q, E_{\text {near }}, E_{\text {far }}\right)=\Delta_{\mathrm{k}}\left[\left\langle f^{2}\right\rangle-\langle f\rangle^{2}\right]+\Delta_{\mathrm{k}}\left[\langle f\rangle^{2}\right] \Delta_{\mathrm{k}} S(Q),
$$

where $\Delta_{\mathrm{k}} S(Q)$ is a differential structure factor, $\alpha_{\mathrm{k}}$ is a normalization constant, and $\Delta_{\mathrm{k}}[\mathrm{]}$ indicates the difference of values in the bracket at $E_{\text {near }}$ and $E_{\text {far }}$ close to an absorption edge of the $k$-th element. $\Delta_{\mathrm{k}} S(Q)$ s are given as a linear combination of partial structures, $S_{\mathrm{ij}}(Q)$, as

$$
\Delta_{\mathrm{k}} S(Q)=\sum_{\mathrm{i}=1}^{\mathrm{N}} \sum_{\mathrm{j}=1}^{\mathrm{N}} W_{\mathrm{ij}}\left(Q, E_{\mathrm{near}}, E_{\mathrm{far}}\right) S_{\mathrm{ij}}(Q)
$$

Here, weighting factors, $W_{\mathrm{ij}}$, are given by

$$
W_{\mathrm{ij}}\left(Q, E_{\text {near }}, E_{\text {far }}\right)=x_{\mathrm{i}} x_{\mathrm{j}} \frac{\Delta_{\mathrm{k}}\left[f_{\mathrm{i}} f_{\mathrm{j}}\right]}{\Delta_{\mathrm{k}}\left[\langle f\rangle^{2}\right]}
$$

where $x_{\mathrm{i}}$ is the atomic concentration of the $i$-th element.

For AXS data analysis, Sasaki's theoretical values ${ }^{30}$ were used for the anomalous terms, $f^{\prime}$ and $f^{\prime \prime}$. The values in Eq. (2) concerning $f$ were calculated with these anomalous terms together with theoretical $f_{0}$ values. $^{31} \quad$ Following a procedure given in Ref. 32, $\Delta_{\mathrm{k}} S(Q)$ spectra were calculated by using Eqs. (1) and (2). The $W_{\mathrm{ij}}$ values at the first peak position of $Q$ $\sim 20 \mathrm{~nm}^{-1}$ are tabulated in Table 1 . They slightly vary with $Q$. Bold digits indicate highly enhanced values in $\Delta_{\mathrm{k}} S(Q)$ compared with those in $S(Q)$. As expected above, the edge-related $S_{\mathrm{ij}}(Q)$ functions are enhanced and other partials are highly suppressed. 
Table 1 The $W_{\mathrm{ij}}$ values of $S_{\mathrm{ij}}(Q)$ for GST (upper) and CGT (lower) at $Q \sim 20 \mathrm{~nm}^{-1}$ (After Refs. 23 and 24)

\begin{tabular}{|c|c|c|c|c|c|c|}
\hline$i-j$ & $\mathrm{Ge}-\mathrm{Ge}$ & $\mathrm{Ge}-\mathrm{Sb}$ & $\mathrm{Ge}-\mathrm{Te}$ & $\mathrm{Sb}-\mathrm{Sb}$ & $\mathrm{Sb}-\mathrm{Te}$ & $\mathrm{Te}-\mathrm{Te}$ \\
\hline$S(Q)$ & 0.026 & 0.075 & 0.167 & 0.054 & 0.281 & 0.367 \\
\hline$\Delta_{\mathrm{Ge}} S(Q)$ & 0.125 & 0.243 & 0.608 & 0.002 & 0.010 & 0.012 \\
\hline$\Delta_{\mathrm{Sb}} S(Q)$ & 0.000 & 0.136 & 0.028 & 0.191 & 0.545 & 0.100 \\
\hline$\Delta_{\mathrm{Te}} S(Q)$ & 0.000 & -0.008 & 0.176 & -0.013 & 0.232 & 0.613 \\
\hline$i-j$ & $\mathrm{Cu}-\mathrm{Cu}$ & $\mathrm{Cu}-\mathrm{Ge}$ & $\mathrm{Cu}-\mathrm{Te}$ & $\mathrm{Ge}-\mathrm{Ge}$ & $\mathrm{Ge}-\mathrm{Te}$ & $\mathrm{Te}-\mathrm{Te}$ \\
\hline$S(Q)$ & 0.065 & 0.070 & 0.309 & 0.019 & 0.168 & 0.369 \\
\hline$\Delta_{\mathrm{Cu}} S(Q)$ & 0.203 & 0.131 & 0.656 & 0.002 & 0.009 & -0.001 \\
\hline$\Delta_{\mathrm{Ge}} S(Q)$ & -0.017 & 0.243 & -0.040 & 0.115 & 0.683 & 0.016 \\
\hline$\Delta_{\mathrm{Te}} S(Q)$ & 0.000 & 0.000 & 0.258 & 0.000 & 0.140 & 0.601 \\
\hline
\end{tabular}

(a)

(i)

(ii) $\alpha_{1}$

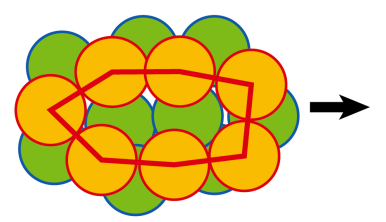

(v) $\alpha_{4}$ (iii) $\alpha_{2}$

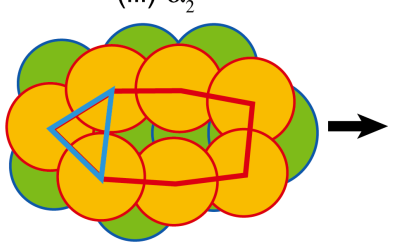

(vi) $\alpha_{5}$

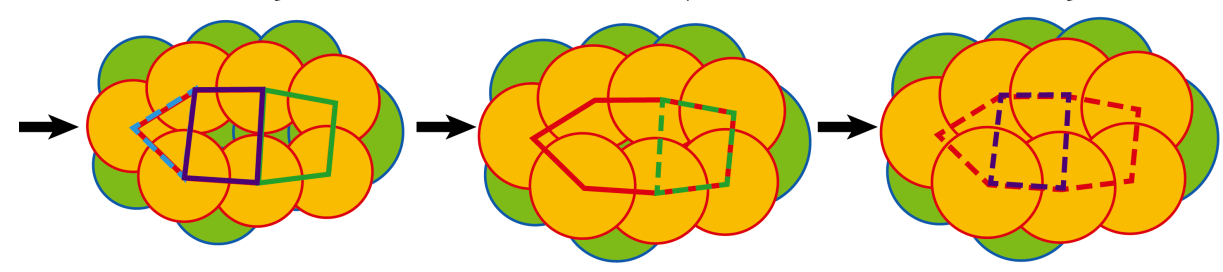

(b)

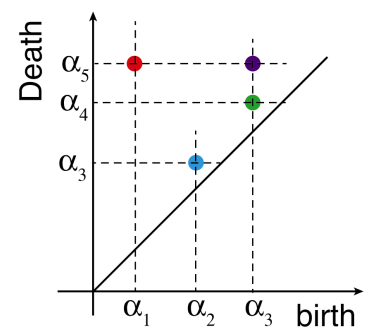

Fig. 2 (a) Schematic view of persistent homology with increasing $\alpha$, and the corresponding (b) persistence diagram.

\subsection{Reverse Monte Carlo modeling}

RMC modeling ${ }^{33}$ is a useful tool to construct 3D structural models of structurally disordered materials using experimental diffraction data. In RMC modeling, atoms of an initial configuration are moved so as to minimize deviations from experimental structural data, e.g., in these studies, two $\Delta_{\mathrm{k}} S(Q) \mathrm{s}$ and one total $S(Q)$, using a standard Metropolis Monte Carlo algorithm. ${ }^{34}$

A starting configuration of a system containing 13500 (GST) or 10000 (CGT) atoms with the corresponding number density was generated using hard-sphere Monte Carlo simulation (random configuration). To avoid unphysical atomic configurations, a constraint of shortest atomic distances is applied: $0.23,0.23,0.20,0.23,0.20$, and $0.30 \mathrm{~nm}$ for $\mathrm{Ge}-\mathrm{Ge}$, $\mathrm{Ge}-\mathrm{Sb}, \mathrm{Ge}-\mathrm{Te}, \mathrm{Sb}-\mathrm{Sb}, \mathrm{Sb}-\mathrm{Te}$, and Te-Te atomic pairs in GST, and $0.245,0.235,0.235,0.235,0.235$, and $0.245 \mathrm{~nm}$ for $\mathrm{Cu}-\mathrm{Cu}$, $\mathrm{Cu}-\mathrm{Ge}, \mathrm{Cu}-\mathrm{Te}$, Ge-Ge, Ge-Te, and $\mathrm{Te}-\mathrm{Te}$ pairs in CGT, respectively. These distances were chosen near values for respective sums of covalent radii, ${ }^{35}$ and were adjusted to fit first coordination shells adequately. The RMC simulations were carried out by using the $\mathrm{RMC}++^{36}$ or $\mathrm{RMC}-\mathrm{POT}^{37}$ program package coded by Gereben et al.

\subsection{Persistent homology analysis}

Persistent homology ${ }^{38}$ is a topological method that can extract hierarchical structures of various non-crystalline solids in the short, medium, and even longer ranges. This method is a mathematical tool for capturing shapes of multiscale data. Figure 2(a) shows a schematic view of persistent homology. The input to the PDs is (i) atomic positions and input radius for each atom, $r_{\mathrm{i}}$. For simplicity, the system is two dimensional and is made of two components, A and B. To characterize the multiscale properties, a parameter is introduced to generate a family of atomic balls having the radius of $r_{\mathrm{i}}(\alpha)=\sqrt{\alpha+r_{\mathrm{i}}^{2}}$. 
For simplicity, $r_{\mathrm{i}}=0$ was set. With varying radii of atomic balls by changing $\alpha$, rings are detected at each $\alpha$. For example, with increasing $\alpha$, a ring of A element (in this case, seven-membered ring given by solid lines) appears at (ii) $\alpha=\alpha_{1}$ in this atomic ball model. With the further increase of $\alpha$, a new threemembered ring appears at (iii) $\alpha=\alpha_{2}$, two four-membered rings appear and the three-membered ring disappears (there becomes no gap as indicated by dashed lines) at (iv) $\alpha=\alpha_{3}$, a fourmembered ring disappears at (v) $\alpha=\alpha_{4}$, and finally the other four-membered ring and the firstly appeared seven-membered ring disappear.

In general, there is an $\alpha=b_{\mathrm{k}}\left(\alpha=d_{\mathrm{k}}\right)$ value at which a ring $k$ first appears (disappears) in the atomic ball model. The $b_{\mathrm{k}}$ and $d_{\mathrm{k}}$ values are called the birth and death scales of the ring $k$, respectively, and describes a map as shown in Fig. 2(b), which is referred to as a persistence diagram (PD). From this construction, $\left(b_{\mathrm{k}}, d_{\mathrm{k}}\right)$ encodes certain scales of each ring, i.e., $b_{\mathrm{k}}$ indicates the maximum distance between two adjacent atoms in the ring, whereas $d_{\mathrm{k}}$ represents the size of ring. The output is expressed as two-dimensional histograms of $b_{\mathrm{k}}$ and $d_{\mathrm{k}}$.

There are two types of persistent homology analysis at present. One is counting ring structures named D1, and another is counting three dimensional cavities named D2. Only the ring structure D1 is present in this paper. Details of the PD analysis are given elsewhere, ${ }^{38}$ and its various applications are described in Ref. 39.

\section{Results and Discussion}

In this section, we introduce experimental results and discussion on two amorphous PCMs, a usual $\mathrm{Ge}_{2} \mathrm{Sb}_{2} \mathrm{Te}_{5}$ (Ref. 23) and an exotic $\mathrm{Cu}_{2} \mathrm{GeTe}_{3}$ (Ref. 24), by investigating partial structures by using AXS experiments close to $K$ edges of constituent elements and RMC modeling.

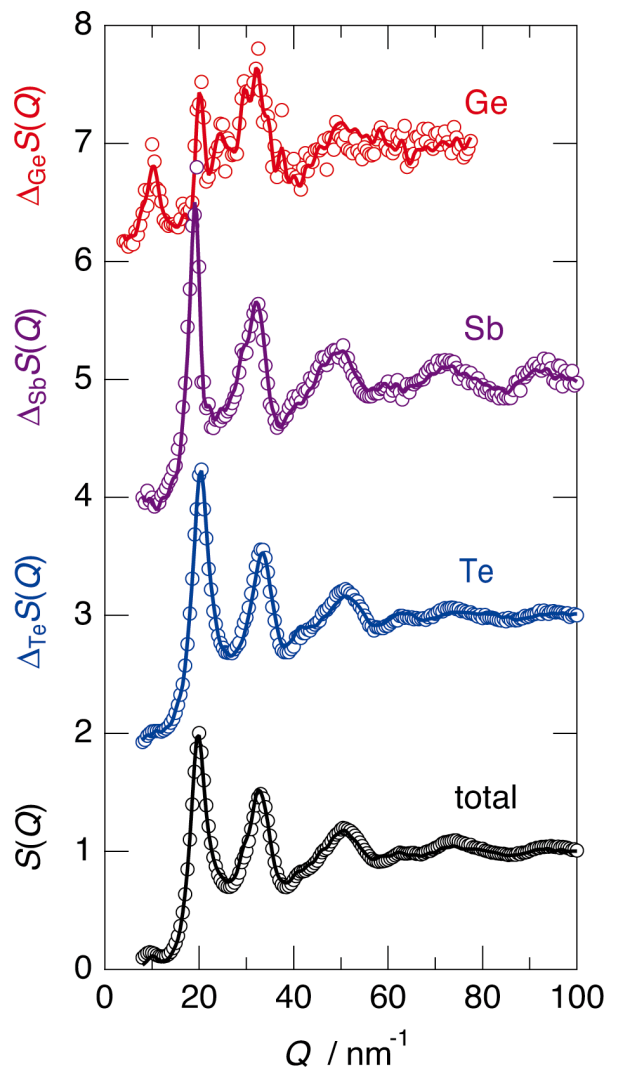

Fig. $3 \Delta_{\mathrm{k}} S(Q)$ s of amorphous GST close to the Ge (red), Sb (purple), and Te (blue) $K$ edges together with $S(Q)$ (black). Solid curves indicate the best fits of the RMC modeling analysis. For clarity, the spectra are displaced upward by 2. After Ref. 23.
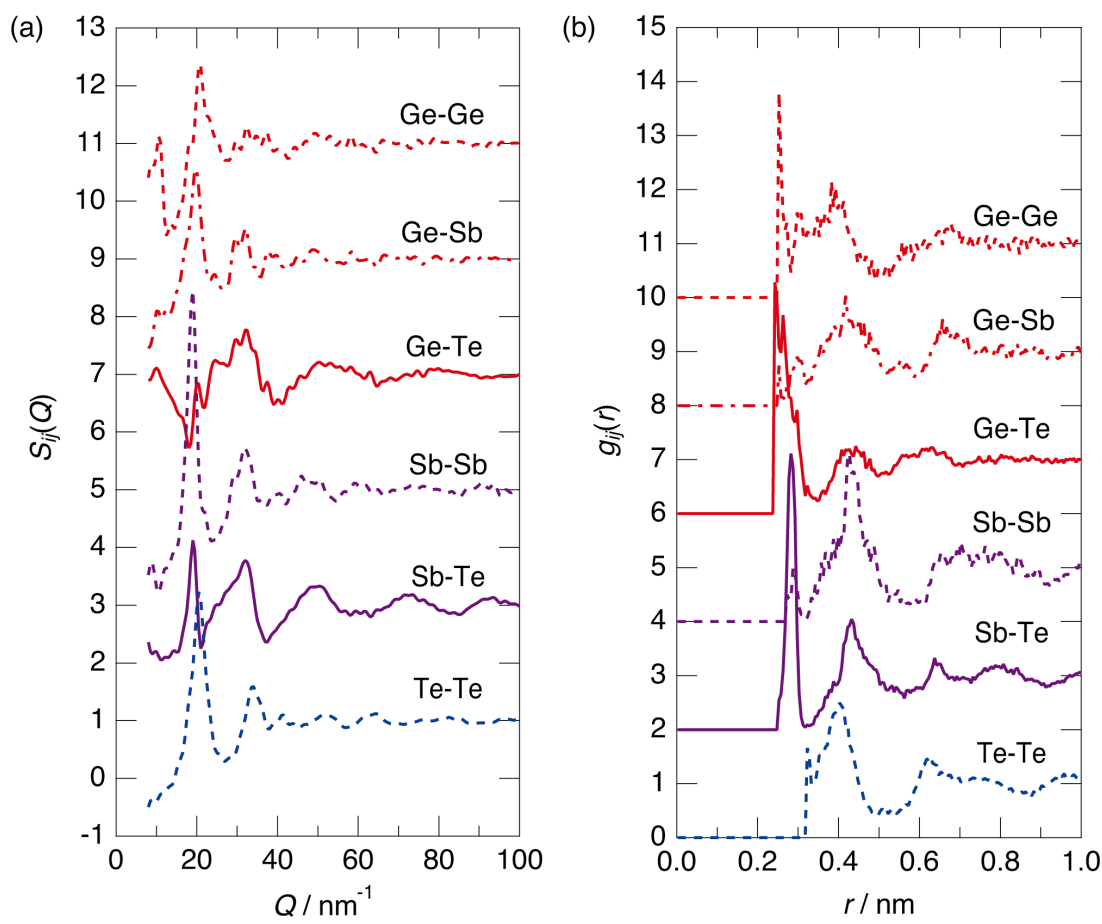

Fig. 4 (a) $S_{\mathrm{ij}}(Q) \mathrm{s}$ and (b) $g_{\mathrm{ij}}(r) \mathrm{s}$ of amorphous GST obtained from the present RMC analysis for the AXS data. After Ref. 23. 
Table 2 The $r_{\mathrm{ij}}$ values for GST (upper) and CGT (lower) together with previous experimental and theoretical results (After Refs. 23 and 24)

\begin{tabular}{|c|c|c|c|c|c|c|}
\hline$i-j$ & $\mathrm{Ge}-\mathrm{Ge}$ & $\mathrm{Ge}-\mathrm{Sb}$ & $\mathrm{Ge}-\mathrm{Te}$ & $\mathrm{Sb}-\mathrm{Sb}$ & $\mathrm{Sb}-\mathrm{Te}$ & $\mathrm{Te}-\mathrm{Te}$ \\
\hline Present $^{23}$ & $0.250(10)$ & & $0.265(5)$ & & $0.282(5)$ & \\
\hline $\mathrm{XAFS}^{5}$ & & & $0.261(1)$ & & $0.285(1)$ & \\
\hline $\mathrm{XAFS}^{7}$ & $0.247(3)$ & & $0.263(1)$ & & $0.283(1)$ & \\
\hline $\mathrm{RMC}^{9}$ & $0.248(2)$ & & $0.264(2)$ & & $0.283(2)$ & \\
\hline $\mathrm{DFT}^{10}$ & & & 0.278 & & 0.293 & \\
\hline DFT/RMC 41 & 0.245 & & 0.275 & & 0.285 & \\
\hline $\mathrm{AIMD}^{11}$ & & & 0.270 & & 0.282 & \\
\hline $\mathrm{AIMD}^{42}$ & & & 0.279 & & 0.294 & \\
\hline$i-j$ & $\mathrm{Cu}-\mathrm{Cu}$ & $\mathrm{Cu}-\mathrm{Ge}$ & $\mathrm{Cu}-\mathrm{Te}$ & $\mathrm{Ge}-\mathrm{Ge}$ & Ge-Te & Te-Te \\
\hline Present $^{24}$ & $0.270(3)$ & $0.271(3)$ & $0.258(2)$ & $0.247(3)$ & $0.255(2)$ & $0.268(2)$ \\
\hline $\mathrm{XAFS}^{20}$ & 0.260 & 0.257 & 0.258 & & 0.260 & \\
\hline $\mathrm{RMC}^{18}$ & $0.258(3)$ & - & $0.255(2)$ & $0.248(2)$ & $0.261(2)$ & $0.275(2)$ \\
\hline AIMD $^{21}$ & 0.259 & 0.251 & 0.263 & 0.296 & 0.280 & 0.298 \\
\hline AIMD $^{22}$ & 0.256 & 0.248 & 0.260 & 0.260 & 0.278 & 0.300 \\
\hline
\end{tabular}

Table 3 The $N_{\mathrm{ij}}$ and $\left\langle N_{\mathrm{i}}\right\rangle$ values for GST (upper) and CGT (lower) together with previous experimental and theoretical results (After Refs. 23 and 24)

\begin{tabular}{|c|c|c|c|c|c|c|c|c|c|c|c|c|}
\hline & $\left\langle N_{\mathrm{Ge}}\right\rangle$ & $N_{\mathrm{GeGe}}$ & $N_{\mathrm{GeSb}}$ & $N_{\mathrm{GeTe}}$ & $\left\langle N_{\mathrm{Sb}}\right\rangle$ & $N_{\mathrm{SbGe}}$ & $N_{\mathrm{SbSb}}$ & $N_{\mathrm{SbTe}}$ & $\left\langle N_{\mathrm{Te}}\right\rangle$ & $N_{\mathrm{TeGe}}$ & $N_{\mathrm{TeSb}}$ & $N_{\mathrm{TeTe}}$ \\
\hline Present $^{23}$ & 4.24 & 0.70 & & 3.26 & 2.95 & & & 2.51 & 2.30 & 1.30 & 1.00 & \\
\hline $\mathrm{XAFS}^{7}$ & & 0.6 & & 3.3 & & & & 2.8 & & 1.2 & 1.2 & \\
\hline $\mathrm{RMC}^{8}$ & 3.7 & & & & 3.0 & & & & & & & \\
\hline $\mathrm{RMC}^{9}$ & 4.24 & 0.69 & & & 3.22 & & & & 2.04 & 1.08 & 0.96 & \\
\hline $\mathrm{DFT}^{10}$ & 4.2 & 0.4 & & & 3.7 & & & & 2.9 & & & \\
\hline DFT/RMC ${ }^{41}$ & 3.92 & 0.36 & & 3.35 & 3.41 & & & 2.65 & 2.56 & 1.33 & 1.06 & \\
\hline AIMD $^{11}$ & $\sim 4$ & & & & $\sim 3$ & & & & $\sim 2$ & & & \\
\hline \multirow[t]{2}{*}{$\mathrm{AIMD}^{42}$} & 3.823 & 0.275 & & 3.277 & 4.025 & & & 3.166 & 2.866 & 1.311 & 1.267 & \\
\hline & $\left\langle N_{\mathrm{Cu}}\right\rangle$ & $N_{\mathrm{CuCu}}$ & $N_{\mathrm{CuGe}}$ & $N_{\mathrm{CuTe}}$ & $\left\langle N_{\mathrm{Ge}}\right\rangle$ & $N_{\mathrm{GeCu}}$ & $N_{\mathrm{GeGe}}$ & $N_{\mathrm{GeTe}}$ & $\left\langle N_{\mathrm{Te}}\right\rangle$ & $N_{\mathrm{TeCu}}$ & $N_{\mathrm{TeGe}}$ & $N_{\mathrm{TeTe}}$ \\
\hline Present $^{24}$ & 5.40 & 2.38 & 0.73 & 2.29 & 4.01 & 1.46 & 0.72 & 1.83 & 4.64 & 1.52 & 0.93 & 2.19 \\
\hline $\mathrm{XAFS}^{20}$ & 4.29 & 2.44 & - & 1.85 & 4.78 & 0.79 & - & 3.99 & & & & \\
\hline $\mathrm{RMC}^{18}$ & $4.1(6)$ & $2.2(4)$ & - & $1.9(3)$ & $4.0(7)$ & - & $1.5(4)$ & $2.5(5)$ & $4.1(5)$ & $1.4(2)$ & $1.0(2)$ & $1.7(3)$ \\
\hline AIMD $^{21}$ & 6.67 & 2.34 & 0.62 & 3.70 & 4.47 & 1.25 & 0.12 & 3.09 & 4.18 & 2.48 & 1.09 & 0.60 \\
\hline $\mathrm{AIMD}^{22}$ & 5.751 & 0.289 & 1.860 & 3.602 & 3.684 & 0.578 & 0.377 & 2.729 & 3.659 & 2.401 & 0.910 & 0.348 \\
\hline
\end{tabular}

\section{4-1 Ordinary PCM: Amorphous $\mathrm{Ge}_{2} \mathrm{Sb}_{2} \mathrm{Te}_{5}$}

Circles in Fig. 3 show $\Delta_{\mathrm{k}} S(Q)$ s of amorphous GST close to the $\mathrm{Ge}$ (red), Sb (purple), and Te (blue) $K$ edges together with $S(Q)$ (black). These functions already indicate some interesting features. A small prepeak is observed in $S(Q)$ at about $Q=$ $10 \mathrm{~nm}^{-1}$, and $\Delta_{\mathrm{Ge}} S(Q)$ has a prominent peak there, while $\Delta_{\mathrm{Sb}} S(Q)$ and $\Delta_{\mathrm{Te}} S(Q)$ show only small peaks similar to $S(Q)$. Thus, the intermediate-range correlations mainly originate from the $\mathrm{Ge}$ related atomic pairs. Also, ${ }_{\mathrm{Ge}} S(Q)$ provides only a small contribution to the distinct first maximum of $S(Q)$ at about $Q=20 \mathrm{~nm}^{-1}$. On the other hand, $\Delta_{\mathrm{Sb}} S(Q)$ and $\Delta_{\mathrm{Te}} S(Q)$ are very similar to $S(Q)$. Therefore, it is no doubt that atomic arrangements around the Ge atoms are considerably different from those around the $\mathrm{Sb}$ and $\mathrm{Te}$ atoms. Solid curves in Fig. 3 show the best fits of the RMC modeling, which coincide very well with the experimental data.

Figure 4(a) shows $S_{\mathrm{ij}}(Q)$ s of amorphous GST obtained from the present RMC analysis for the AXS data. The features of the $\mathrm{Sb}-\mathrm{Sb}$ and Te-Te partials are basically similar to each other, indicating similar local environments around the $\mathrm{Sb}$ and $\mathrm{Te}$ atoms as in the metastable rock salt crystal. Ge-Te related partials are very different from each other, and resemble well those of $\mathrm{GeSe}_{2}$ glass. ${ }^{32,40}$ 1) The Ge-Ge correlation shows a prominent prepeak, indicating the existence of intermediaterange order; 2) the Ge-chalcogen partial structure has a minimum at the first maximum in $S(Q)$. Therefore, the amorphous structure around the Ge atoms is expected to be very different from the three- or six-coordinated crystal.

Figure 4(b) shows $g_{\mathrm{ij}}(r) \mathrm{s}$ of amorphous GST obtained from the present RMC analysis for the AXS data. The overall features are similar to results of a DFT calculation ${ }^{41}$ and an $a b$ initio MD (AIMD) simulation. ${ }^{42}$ The partial nearest neighbor distances, $r_{\mathrm{ij}}$, obtained from $g_{\mathrm{ij}}(r) \mathrm{s}$ are tabulated in Table 2 (upper) together with previous experimental ${ }^{5,7,9}$ and theoretical ${ }^{10,11,41,42}$ results.

Around the $\mathrm{Ge}$ atoms, heteropolar Ge-Te bonds are centered at about $0.265 \mathrm{~nm}$, in good agreement with previous experimental results ${ }^{5,79}$ and shorter than theoretical data. ${ }^{10,11,41,42}$ In addition to the $\mathrm{Ge}-\mathrm{Te}$ bonds, $\mathrm{Ge}-\mathrm{Ge}$ wrong homopolar bonds are seen at about $0.25 \mathrm{~nm}$, again in good agreement with previous experimental results. ${ }^{41,42}$ Around the $\mathrm{Sb}$ atoms, $\mathrm{Sb}-\mathrm{Te}$ heteropolar bonds are mainly coordinated at about $0.283 \mathrm{~nm}$, in good agreement with all of the previous experimental results ${ }^{5,7,9}$ and most of the theoretical ones. ${ }^{11,41}$

Average partial coordination numbers of $j$ th atoms around the $i$ th atom, $N_{\mathrm{ij}}$, and $\left\langle N_{\mathrm{i}}\right\rangle$ values are listed in Table 3 (upper) together with previous experimental ${ }^{7-9}$ and theoretical ${ }^{10,11,41,42}$ 


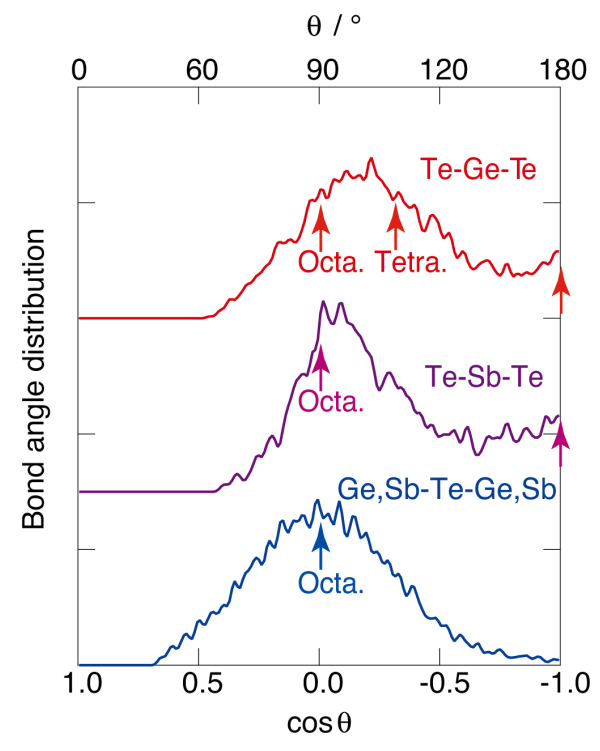

Fig. 5 Bond angle distributions of heteropolar bonds of amorphous GST around the Ge (red), Sb (purple), and Te (blue) atoms obtained from the present AXS study. After Ref. 23.

results. $\left\langle N_{\mathrm{Ge}}\right\rangle$ is 4.24 , almost following the $8-N$ rule. About 0.70 are composed of the wrong Ge-Ge bonds. $\left\langle N_{\mathrm{Sb}}\right\rangle$ is 2.95 , again following the octet rule. This result is in good agreement with other experimental data, ${ }^{7-9}$ while the theories mostly overestimated. ${ }^{10,41,42}$ Most $\mathrm{Sb}$ atoms are surrounded by Te atoms $\left(N_{\mathrm{SbTe}}=2.51\right) .\left\langle N_{\mathrm{Te}}\right\rangle$ is 2.30 , exceeding the $8-N$ rule of two, unlike in previous experimental ${ }^{9}$ and theoretical ${ }^{11}$ works.

Bond angle distributions around Ge are the most controversial issue on the intermediate-range structures of amorphous GST. Kolobov et al. proposed an umbrella flip model, in which Ge atoms have tetrahedral configurations of $109^{\circ} .5$ On the contrary, a single peak was observed at an angle slightly larger than $90^{\circ}$ in an RMC result by Kohara et al. ${ }^{8}$ and a DFT one by Akola and Jones. ${ }^{10}$ From these results, they emphasized that a significant number of fragments of the metastable rock salt crystal are contained in amorphous GST. On the other hand, Akola and Jones $^{10}$ also pointed out that the peak is broad and includes tetrahedral symmetry, which becomes prominent when the Ge-Te bonds are defined to be shorter. Furthermore, double peaks were presented by ab initio MD results, ${ }^{11}$ in which the octahedral-tetrahedral ratio is about 1.0:0.7. Another ab initio MD result ${ }^{42}$ also shows the existence of tetrahedral symmetry around Ge to be about $33 \%$.

Figure 5 shows the bond angle distributions of heteropolar bonds around the $\mathrm{Ge}$ (red), Sb (purple), and Te (blue) atoms obtained from the present AXS study for amorphous GST. Around $\mathrm{Ge}$, the spectrum is widely distributed and seems to have two peaks centered at 90 and $109^{\circ}$, suitable for octahedral and tetrahedral atomic configurations, respectively. The ratio can roughly be estimated to be 50:50.

Figure 6(a) shows atomic configurations of Ge (middle red), $\mathrm{Sb}$ (small purple), and Te (large blue) atoms in amorphous GST obtained from the RMC modeling, where the thickness is $1 / 3$ of the width for the clarity. Around Ge, they are mainly composed of tetrahedral units, where $\mathrm{GeTe}_{4}$ and $\mathrm{GeGeTe}_{3}$ tetrahedra coexist. Around $\mathrm{Sb}$, they are mainly $\mathrm{SbTe}_{3}$ pyramidal units together with a small portion of T-shape configurations. More detailed figures are given in Figs. 5(b) and 5(c) of Ref. 23.

Figure 6(b) shows only the square rings extracted from (a)

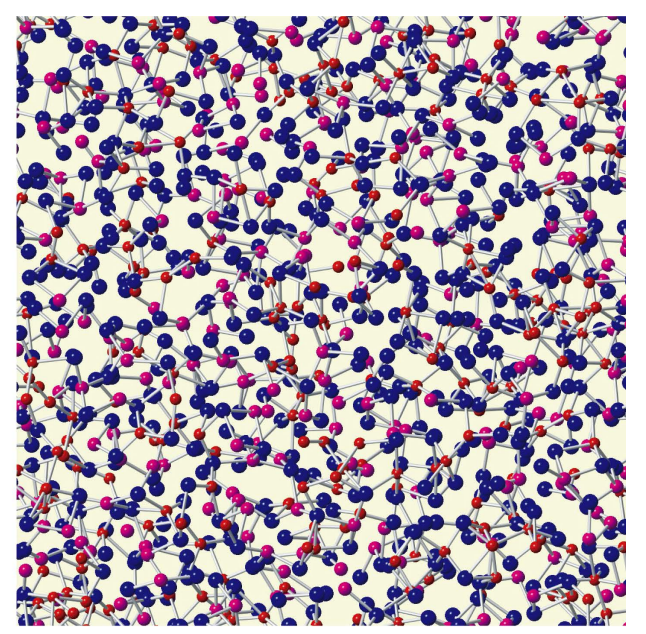

(b)

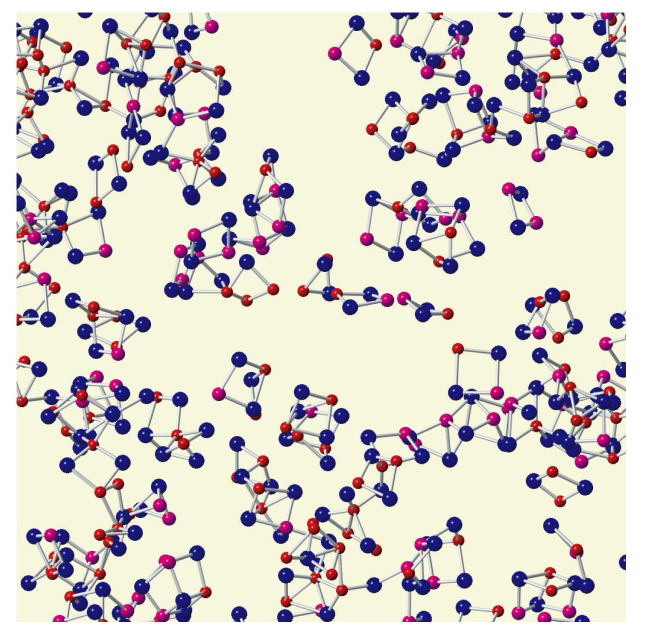

Fig. 6 (a) Atomic configurations of Ge (middle red), Sb (small purple), and Te (large blue) atoms in amorphous GST obtained from the RMC modeling. (b) Only the square rings extracted from (a). After Ref. 23.

Fig. 5(a). About $40 \%$ of the constituent atoms belong to the square rings. Note that more than $50 \%$ of Ge atoms are the member of square rings, while only about $30 \%$ of $\mathrm{Sb}$ atoms contribute the square rings. Thus, the square rings are preferably made up of $\mathrm{Ge}-\mathrm{Te}-\mathrm{Ge}-\mathrm{Te}$, which was not pointed out by the theories. ${ }^{10,11}$ It should also be noted that the shapes of the square rings are mostly highly puckered, unlike the illustrations in Refs. 10 and 11.

Structurally, puckered and unpuckered square rings are topologically the same. However, this slight structural change may largely affect the electronic structures. Huang and Robertson $^{43}$ pointed out that the optical matrix elements are enhanced in the crystal by aligned rows of resonantly bonded $p$ orbitals. By the formation of puckered square rings on the crystalline-amorphous phase change, the $p$ orbitals loose the directional order even inside the square rings, which should induce significant changes in the electronic properties.

Figure 7 shows the persistence diagrams, i.e., the birth-death map obtained from the persistent homology analysis, of amorphous GST for the ring structure around (a) Ge, (b) Sb, (c) $\mathrm{Ge}$ and $\mathrm{Sb}$, and (d) Te elements. As shown in (a) concerning $\mathrm{Ge}$, the birth-death points are mostly distributed just above the diagonal slope line, showing a typical feature of random distribution of Ge atoms, which was examined by using a Lenard-Jones glassy system in Fig. 7 of Ref. 38 . In (b) 
(a) $\mathrm{Ge}$

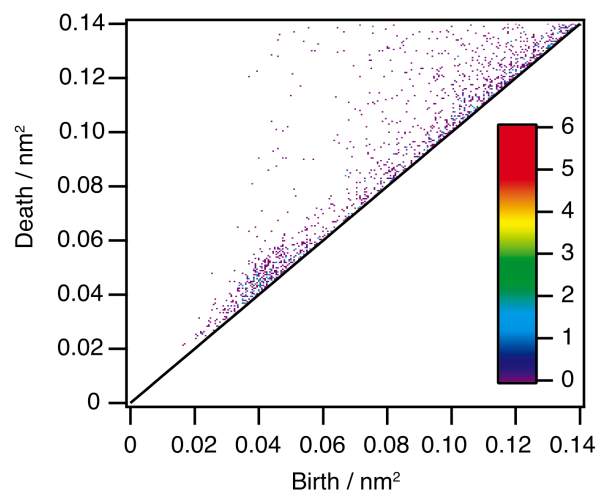

(c) $\mathrm{Ge} \& \mathrm{Sb}$

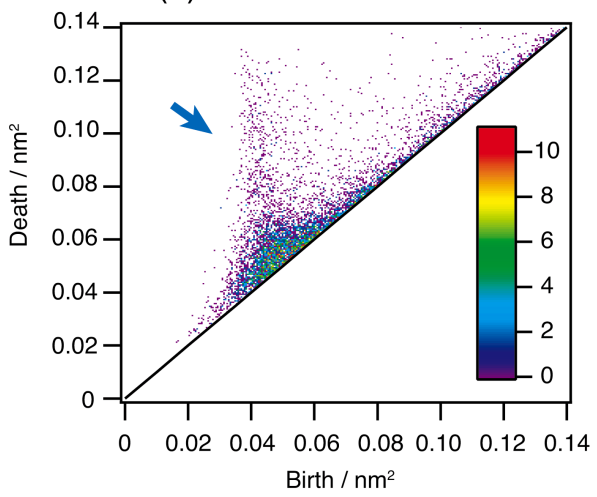

(b) $\mathrm{Sb}$

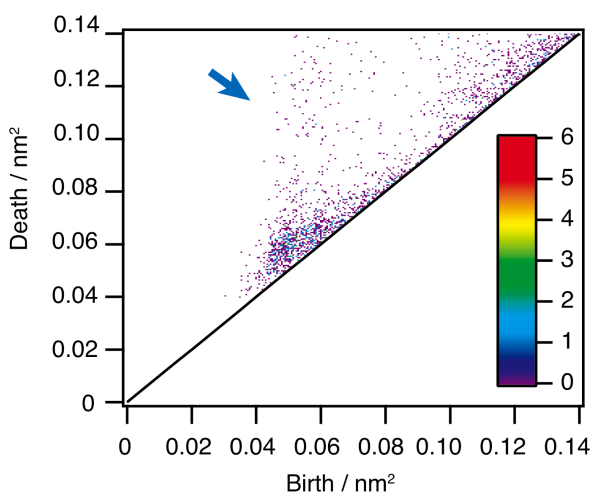

(d) $\mathrm{Te}$

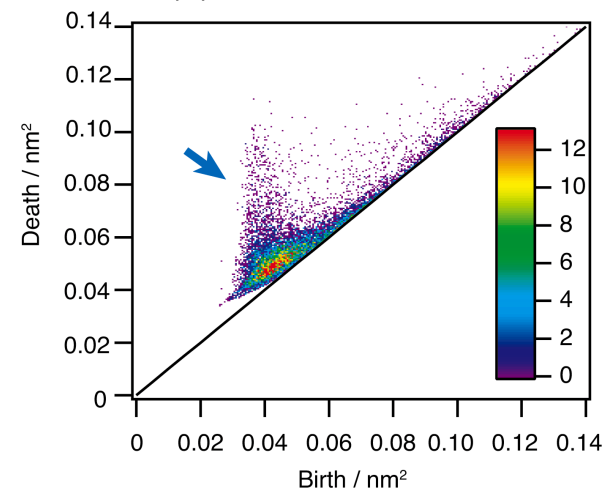

Fig. 7 The persistence diagrams of amorphous GST for the ring structure $\mathrm{D}_{1}$ concerning (a) Ge, (b) $\mathrm{Sb}$, (c) Ge and Sb, and (d) Te elements.

concerning Sb, a small contribution appears in the area of longdeath in the birth range of $0.04-0.06 \mathrm{~nm}^{2}$ as shown by the arrow, indicating the existence of large rings with $\mathrm{Sb}$ atoms with a diameter of $0.5-0.7 \mathrm{~nm}$. When the $\mathrm{Ge}$ and $\mathrm{Sb}$ atoms are combined for the persistent homology analysis, a larger number of large rings with the similar size are found as shown by the arrow in (c). In (d) concerning Te, large rings with Te atoms are also observed as shown by the arrow, while the death range is smaller, indicating a smaller ring size of less than $0.5 \mathrm{~nm}$.

In summary, short- and intermediate-range local atomic structures of amorphous GST were investigated by a combination of AXS experiments and RMC analysis for clarifying the fast crystalline-amorphous phase change process and long lifetime as a storage material. From the obtained atomic configurations, it was found that the $\mathrm{Sb}$ atoms and about a half of the Ge atoms have octahedral environments similar to those in the crystal, which may play roles in the fast phase change process. The remaining half of the Ge atoms with the tetrahedral symmetry act as a proper energy barrier between the phases, giving a long lifetime of amorphous GST. A large number of puckered square rings result in highly disordered $p$ electron directions, indicating a significant reduction of the resonant bonds and optical matrix elements in the amorphous phase. The persistent homology analysis revealed the existence of large-size $\mathrm{Ge} / \mathrm{Sb}$ rings and smaller-size $\mathrm{Te}$ rings in the intermediate-range atomic configurations, which may give a hint for the further structural analysis in amorphous GST.

\section{4-2 Exotic PCM: Amorphous $\mathrm{Cu}_{2} \mathrm{GeTe}_{3}$}

Since CGT shows an exotic nature in its electronic and thermodynamic properties, i.e., anomalous behavior in optical reflectance and density on the crystalline-amorphous phase change, a different structural feature is highly expected in contrast to GST. Circles in Fig. 8 show $\Delta_{\mathrm{k}} S(Q)$ s of amorphous CGT close to the $\mathrm{Cu}$ (red), Ge (purple), and Te (blue) $K$ edges together with $S(Q)$ (black). These differential structure factors already indicate some interesting features. A small prepeak is observed at about $10 \mathrm{~nm}^{-1}$ in $\Delta_{\mathrm{Cu}} S(Q)$ and $\Delta_{\mathrm{Ge}} S(Q)$, while such an indication is very small in $\Delta_{\mathrm{Te}} S(Q)$ and $S(Q)$. Thus, intermediate-range correlations mainly originate from $\mathrm{Cu}$ - and Ge-related partials and not from Te ones. $\Delta_{\mathrm{Ge}} S(Q)$ provides a small contribution to the distinct first maximum in $S(Q)$ at about $20 \mathrm{~nm}^{-1}$, while $\Delta_{\mathrm{Te}} S(Q)$ indicates a much larger contribution there. Therefore, it is reasonable to suggest that atomic arrangements around the $\mathrm{Ge}$ atoms are different from those around $\mathrm{Cu}$ and $\mathrm{Te}$ atoms. Solid curves in Fig. 7 show the best fits of the RMC modeling, including XAFS results for the analysis (see Ref. 24 for details), which are again in good agreement with the experimental data.

Figure 9(a) shows $S_{\mathrm{ij}}(Q)$ s of amorphous CGT obtained from the present RMC analysis for the AXS data. Besides the peak heights, all the $S_{\mathrm{ij}}(Q)$ s have basically similar features in contrast to those of GST. In particular, the $\mathrm{Cu}-\mathrm{Te}$ and $\mathrm{Ge}-\mathrm{Te}$ heteropolar correlations are very similar to each other. Only the $\mathrm{Cu}-\mathrm{Cu}$ correlation has a quite large minimum at the first peak position of about $20 \mathrm{~nm}^{-1}$, where the Te-Te contribution is very large.

Figure 9(b) shows $g_{\mathrm{ij}}(r) \mathrm{s}$ of amorphous CGT obtained from the present RMC analysis for the AXS data. The overall features of $g_{\mathrm{ij}}(r) \mathrm{s}$ are similar to one another, i.e., the first and second peaks are located at about 0.26 and $0.42 \mathrm{~nm}$, respectively, although the values slightly change depending on elemental pairs. Note that a considerable number of the homopolar bonds 
are observed in the amorphous phase unlike in the crystal. The $r_{\mathrm{ij}}$ values obtained from $g_{\mathrm{ij}}(r) \mathrm{s}$ are tabulated in Table 2 (lower) together with previous experimental ${ }^{18,20}$ and theoretical ${ }^{1,22}$ results.

Around the $\mathrm{Cu}$ atoms, heteropolar $\mathrm{Cu}-\mathrm{Te}$ bonds are located at about $0.258 \mathrm{~nm}$, in good agreement with previous experimental ${ }^{18,20}$ and theoretical ${ }^{21,22}$ results within the error bars. In addition, $\mathrm{Cu}-\mathrm{Cu}$ and $\mathrm{Cu}-\mathrm{Ge}$ homopolar wrong bonds are both observed at

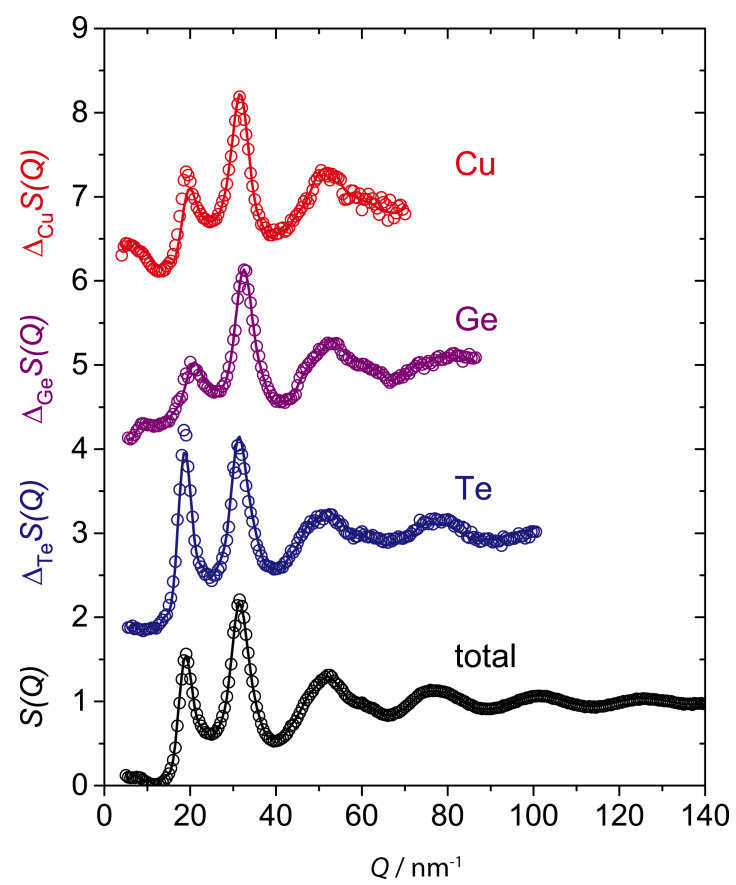

Fig. $8 \Delta_{\mathrm{k}} S(Q)$ s of amorphous CGT close to the $\mathrm{Cu}$ (red), Ge (purple), and Te (blue) $K$ edges together with $S(Q)$ (black). Solid curves indicate the best fits of the RMC modeling analysis. For clarity, the spectra are displaced upward by 2 . After Ref. 24 . about $0.270 \mathrm{~nm}$, which are slightly longer than all of the previous results. Around the $\mathrm{Ge}$ atoms, Ge-Te heteropolar bonds are mainly located at about $0.255 \mathrm{~nm}$, which is very slightly shorter than the experimental results ${ }^{18,20}$ and much shorter than the theoretical ${ }^{21,22}$ data. Ge-Ge homopolar bonds are located at about $0.247 \mathrm{~nm}$, in good agreement with a previous RMC result ${ }^{18}$ and smaller than the theoretical ones. ${ }^{21,22}$ The Te-Te homopolar bond length of $0.268 \mathrm{~nm}$ is shorter than the previous data. ${ }^{18,21,22}$

The $N_{\mathrm{ij}}$ and $\left\langle N_{\mathrm{i}}\right\rangle$ values are tabulated in Table 3 (lower) together with previous experimental ${ }^{18,20}$ and theoretical ${ }^{21,22}$ results. Concerning the $\left\langle N_{\mathrm{i}}\right\rangle$ values, the previous RMC result ${ }^{18}$ emphasized that they are almost the crystal values of 4 around all of the elements. On the other hand, the present results exceed 4 , in particular that around $\mathrm{Cu}$, which is consistent with theoretical results. ${ }^{21,22}$ The reason for the differences was confirmed to be due to the lack of AXS data and too strong constraint of the lack of $\mathrm{Cu}-\mathrm{Ge}$ correlations in the previous RMC analysis. The overall features in $N_{\mathrm{ij}}$ is rather similar to those of an $a b$ initio MD calculation, ${ }^{21}$ although each value does not always coincide with each other.

Figure 10(a) shows bond angle distributions around the $\mathrm{Cu}$ (red), Ge (purple), and Te (blue) atoms with every element obtained from the present AXS study. In general, broad distributions are observed around $109^{\circ}$ for all the correlations, relating to a tetrahedral coordination. This corresponds to a large number of fourfold coordinated atoms, i.e., a largely distorted crystal structure. Besides, it should be noted that large and narrow peaks are observed around $60^{\circ}$, and that of the $\mathrm{Cu}$-centered spectrum is most prominent, which are consistent with the previous theoretical results. ${ }^{21,22}$

These features can be understood by calculating ring statistics, which were calculated using the R.I.N.G.S. program. ${ }^{44}$ The ring was defined as a primitive one, i.e., a closed path of bonds originating from and leading back to the same atom, and the results are shown in Fig. 10(b). A distribution of ring structures is observed with a broad maximum centered at six-membered rings corresponding to the broad $109^{\circ}$-peak in (a), which indicates again a large distortion of the crystal structure (a)

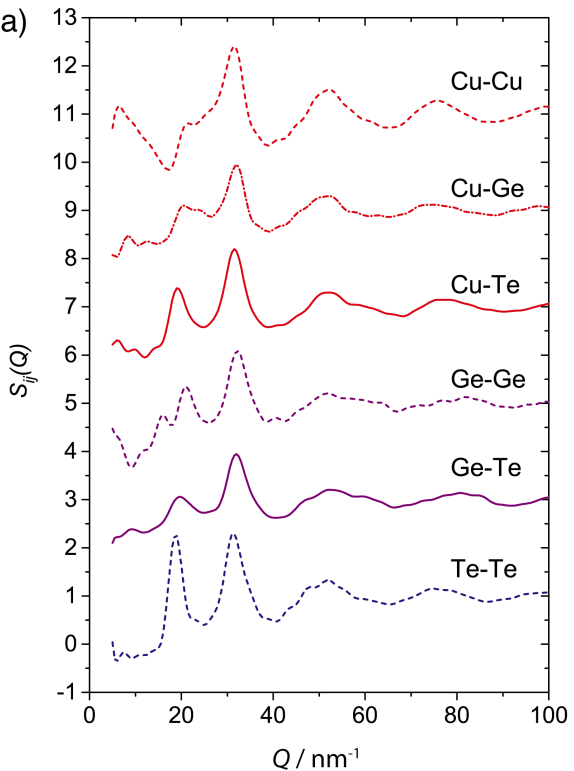

(b)

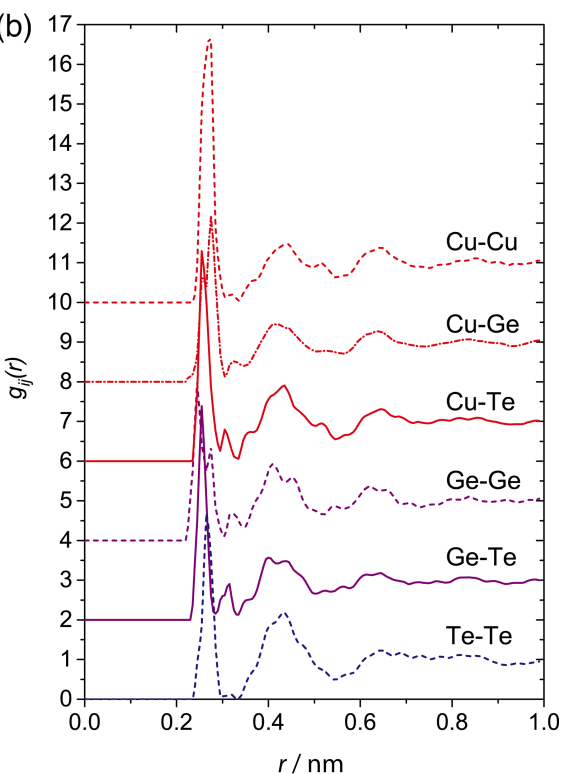

Fig. 9 (a) $S_{\mathrm{ij}}(Q) \mathrm{s}$ and (b) $g_{\mathrm{ij}}(r) \mathrm{s}$ of amorphous CGT obtained from the present RMC analysis for the AXS data. After Ref. 24 

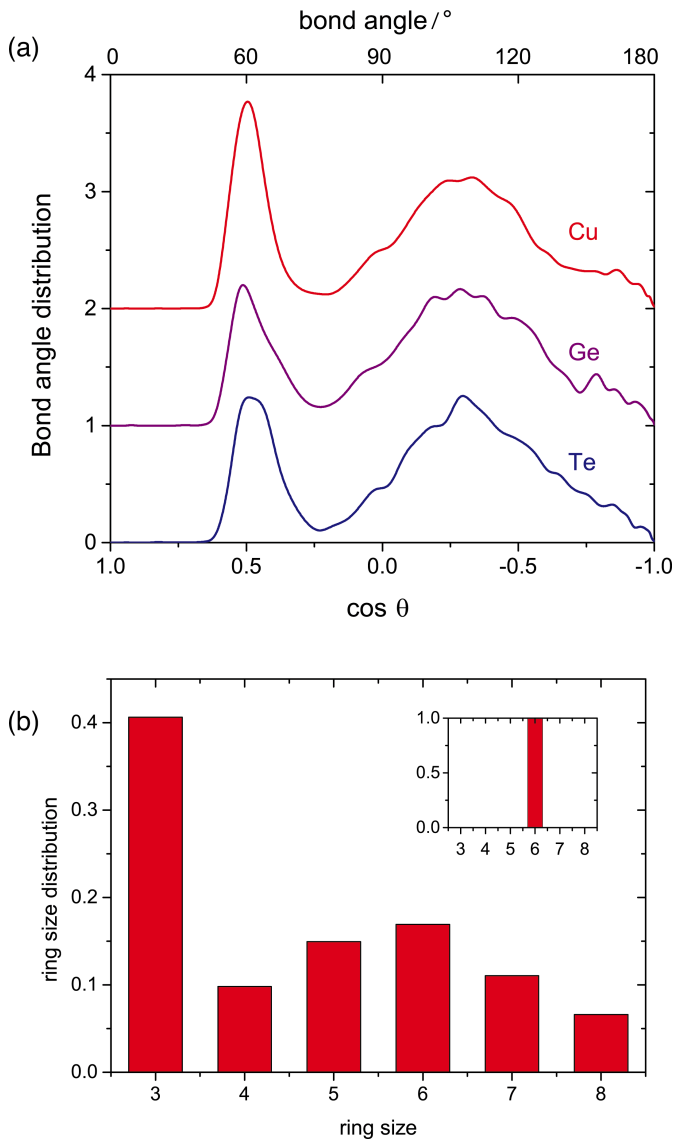

Fig. 10 (a) Bond angle distributions of amorphous CGT around the $\mathrm{Cu}$ (red), $\mathrm{Ge}$ (purple), and $\mathrm{Te}$ (blue) atoms with every elements obtained from the present AXS study. (b) Ring statistics in amorphous CGT. Inset: Those in crystalline CGT. After Ref. 24.

consisting of only six-membered rings (see Fig. 1(b) and the inset of Fig. 10(b).). A large fraction of threefold rings is also realized, corresponding to the sharp peaks around $60^{\circ}$ in (a), which is consistent with the theoretical results..$^{21,22}$

Figure 11(a) shows atomic configurations of $\mathrm{Cu}$ (middle red), Ge (small purple), and Te (large blue) atoms in amorphous CGT obtained from the RMC modeling, where the thickness is $1 / 2$ of the width for the clarity. At a glance, all of the constituent atoms form a random network with coordination numbers of 3 - 5. It should be also noted that concentration fluctuations are observed, in particular, large sized $\mathrm{Cu}$-clusters are seen in some parts in the figure.

Figure 11(b) exhibits only the triangle rings extracted from Fig. 11(a). About $70 \%$ of the constituent atoms belong to the triangle rings. Note that the fractions highly depend on the elements, i.e., 83,70 , and $60 \%$ for $\mathrm{Cu}, \mathrm{Ge}$, and $\mathrm{Te}$, respectively. In fact, a large number of $\mathrm{Cu}_{3}$ rings are observed, in particular, in the $\mathrm{Cu}$-rich region in (a).

In contrast to the structure of crystalline CGT, the sixmembered ring structure collapses upon amorphization to form a smaller-ring structure, and the average coordination numbers increase. Such a crystal-noncrystal change looks very similar to the melting of tetrahedral semiconductors, $\mathrm{Si}, \mathrm{Ge}$, or GaSb. ${ }^{45}$ Note that the density increase and reflectivity increase by metallization happen in these semiconductors on melting by an increase of coordination number, ${ }^{46,47}$ which is also similar to the present transition in CGT, although the electrical conductivity (a)

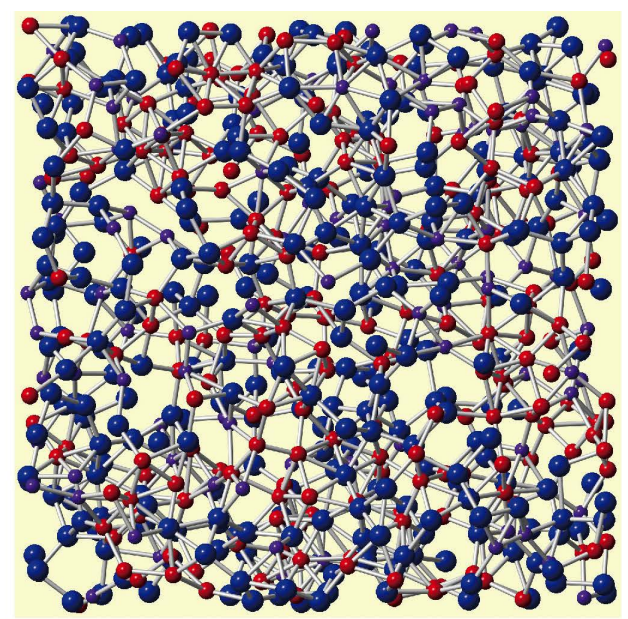

(b)

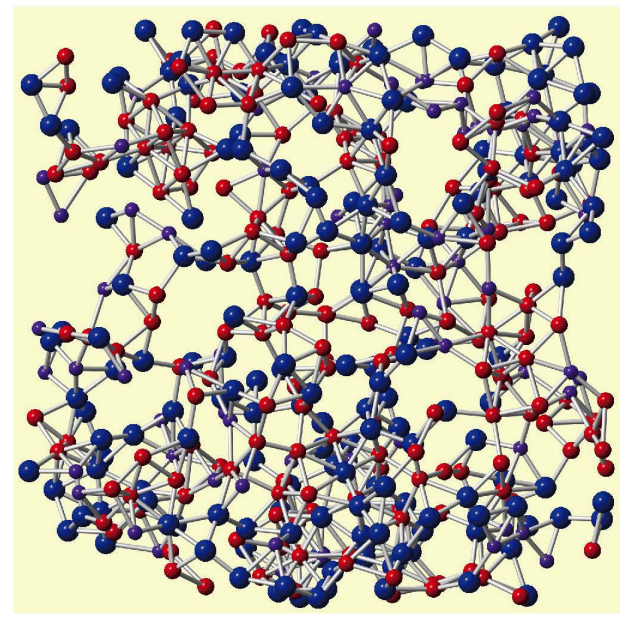

Fig. 11 (a) Atomic configurations of $\mathrm{Cu}$ (middle red), $\mathrm{Ge}$ (small purple), and Te (large blue) atoms in amorphous CGT obtained from the RMC modeling. (b) Only the triangle rings extracted from (a). After Ref. 24.

conflicts. In addition, $\mathrm{Cu}$ atoms should be moved on amorphization to form the $\mathrm{Cu}_{3}$ rings and $\mathrm{Cu}$-rich region. Such movements of $\mathrm{Cu}$ atoms may easily be accomplished due to the superionic nature of $\mathrm{Cu}$ chalcogenide materials. ${ }^{48}$

Figure 12 shows the persistence diagram of amorphous CGT for the ring structure concerning (a) $\mathrm{Cu}$, (b) $\mathrm{Ge}$, (c) $\mathrm{Cu}$ and $\mathrm{Ge}$, and (d) $\mathrm{Te}$ atoms. As shown in (a) concerning $\mathrm{Cu}$, a prominent number of the birth-death points are observed near $0.02 / 0.02 \mathrm{~nm}^{2}$, which corresponds to the $\mathrm{Cu}_{3}$ triangles frequently seen in Fig. 11(b). Besides, small distributions are observed in the area of long-death in the birth range of $0.02 \mathrm{~nm}^{2}$, indicating the existence of large rings consisting of $\mathrm{Cu}$ atoms. This result may suggest the existence of ion conducting pathways of $\mathrm{Cu}$ atoms in amorphous CGT, which is consistent with the abovementioned rapid ionic conduction of $\mathrm{Cu}$.

On the other hand, a typical feature of random distributions is seen with the Ge atoms in (b), where the birth-death points are mostly distributed just above the diagonal slope line. When the $\mathrm{Cu}$ and $\mathrm{Ge}$ atoms are combined for the persistent homology analysis, a larger number of larger rings are observed as shown by the arrow in (c). Therefore, the existence of correlations is confirmed between the $\mathrm{Cu}$ and $\mathrm{Ge}$ (cation) atoms by the appearance of large rings. In (d) concerning Te, large rings with Te are observed as shown by the arrow, while the ring size is 
(a) $\mathrm{Cu}$

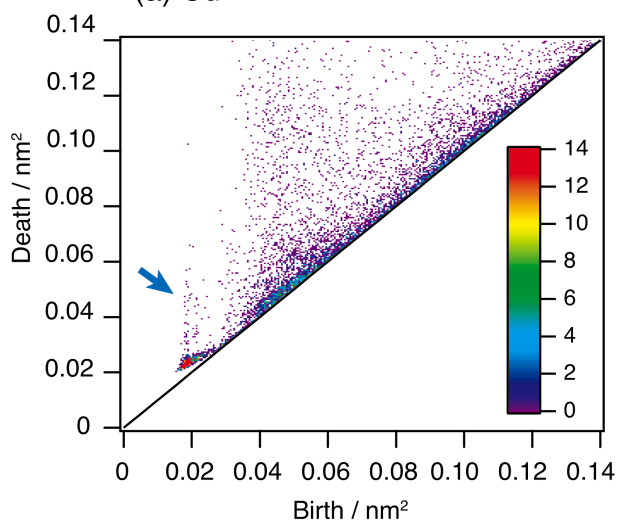

(c) $\mathrm{Cu} \& \mathrm{Ge}$

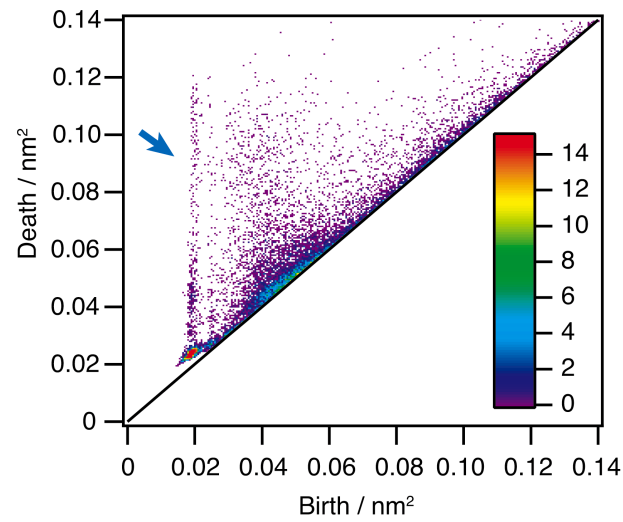

(b) $\mathrm{Ge}$

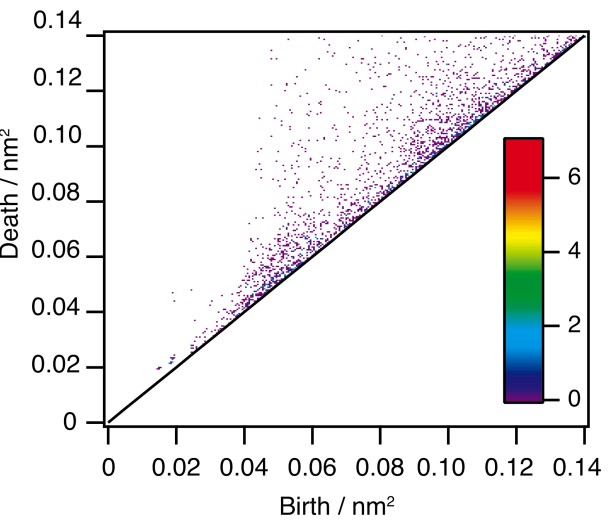

(d) $\mathrm{Te}$

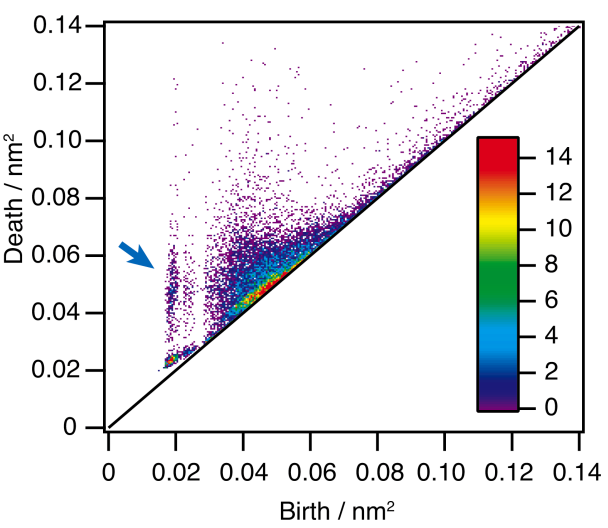

Fig. 12 The persistence diagrams of amorphous CGT for the ring structure $\mathrm{D}_{1}$ concerning (a) $\mathrm{Cu}$, (b) $\mathrm{Ge},(\mathrm{c}) \mathrm{Cu}$ and $\mathrm{Ge}$, and (d) Te elements. After Ref. 24.

again smaller compared with those of the cation rings.

In summary, short- and intermediate-range local atomic structures of amorphous CGT were investigated by a combination of AXS and RMC. A significant number of triangle configurations are observed, in particular with the $\mathrm{Cu}$ atoms. On amorphization, the present experimental results reveal that (1) six-membered rings in the crystal are largely distorted or collapsed into triangles, and (2) rapid movements of the $\mathrm{Cu}$ atoms are highly required.

\section{Conclusions}

In the present studies, a combination of AXS experiments and RMC modeling was confirmed to be a powerful tool to investigate local atomic structures of amorphous materials, in particular, for the intermediate distance range. Information in this range is important to clarify the relationship between the material properties and partial atomic structures of useful functional amorphous materials such as PCMs.

As introduced above in this article, amorphous GST and CGT have different types of intermediate-range atomic structures from each other, although they are categorized into the PCM group, and the structural differences are highly related to the differences of electronic properties upon the crystallineamorphous phase changes. The presence of puckered square rings in the amorphous GST may produce the highly disordered $p$ electron directions, resulting in a significant reduction of resonant bonds and the largely non-metallic nature in amorphous GST. On the other hand, the collapse of the six-membered rings into triangle configurations and the corresponding density decrease induce metallization upon the amorphization of CGT.

The present AXS experiments were mainly carried out at BM02 of the ESRF, which is a typical bending magnet synchrotron radiation source, although the optics for incident $\mathrm{X}$-rays are quite excellent. At the SPring-8, an AXS setup was constructed $^{49}$ at an undulator beamline of BL13XU, where the incident X-ray intensity is much stronger than that of the present works by about two orders of magnitude. Therefore, much more reliable outputs will be expected for several kinds of functional amorphous materials. Furthermore, new fields of research will become available, such as time-resolved AXS experiments on PCMs to trace atomic structures during amorphous-crystalline phase changes in $10 \mathrm{~ns}$ time scale.

\section{Acknowledgements}

We acknowledge Prof. M. Wuttig and Prof. Y. Sutou for providing the amorphous GST and CGT samples, respectively. The AXS experiments were performed with the help of Dr. N. Boudet, Dr. N. Blanc, and Dr. J.-F. Bérar at BM02 of the ESRF (Nos. HD279, HD380, HD466, HD510, HC2213, and HC2534). The supporting AXS experiments were performed at BL13XU of the SPring-8 (Nos. 2014A1624 and 2014B1733). The XAFS experiments were carried out at BL12C of the KEK-PF (Nos. 2010G559 and 2012G522). These works were supported by the Japan Society for the Promotion of Science (JSPS) Grant-in-Aid for Scientific Research on Innovative Areas "3D Active-Site Science" (No. 26105006) and "Sparse Modeling" (No. 
16H01553), and the Japan Science and Technology Agency's (JST) CREST (No. JPMJCR1861). This research was also supported by JST PRESTO (No. JPMPR15N4); the "Materials Research by Information Integration" initiative $\left(\mathrm{MI}^{2} \mathrm{I}\right)$ project of the Support Program for Starting Up Innovation Hub from JST. J. R. S. gratefully acknowledges financial support as Overseas Researcher under a JSPS fellowship (No. P16796).

\section{References}

1. M. Wuttig and N. Yamada, Nat. Mater, 2007, 6, 824.

2. E. Garcia-Garcia, A. Mendoza-Galvan, Y. Vorobiev, E. Morales-Sanchez, J. Gonzalez-Hernandez, G. Martinez, and B. S. Chao, J. Vac. Sci. Technol. A, 1999, 17, 1805.

3. I. Friedrich, V. Weidenhof, W. Njoroge, P. Franz, and M. Wuttig, J. Appl. Phys., 2000, 87, 4130.

4. N. Yamada and T. Matsunaga, J. Appl. Phys., 2000, 88, 7020.

5. A. V. Kolobov, P. Fons, A. I. Frenkel, A. L. Ankudinov, J. Tominaga, and T. Uruga, Nat. Mater, 2004, 3, 703.

6. T. Chattopadhyay, J. X. Boucherle, and H. G. von Schnering, J. Phys. C, 1987, 20, 1431.

7. D. A. Baker, M. A. Paesler, G. Lucovsky, S. C. Agarwal, and P. C. Taylor, Phys. Rev. Lett., 2006, 96, 255501.

8. S. Kohara, K. Kato, S. Kimura, H. Tanaka, T. Usuki, K. Suzuya, H. Tanaka, T. Matsunaga, N. Yamada, Y. Tanaka, H. Suematsu, and M. Takata, Appl. Phys. Lett., 2006, 89, 201910.

9. P. Jóvári, I. Kaban, J. Steiner, B. Beuneu, A. Schöp, and A. Webb, J. Phys.: Condens. Matter, 2007, 19, 335212.

10. J. Akola and R. O. Jones, Phys. Rev. B, 2007, 76, 235201.

11. J. Hegedüs and S. R. Elliott, Nat. Mater., 2008, 7, 399.

12. N. Yamada, E. Ohno, K. Nishiuchi, N. Akahira, and M. Takao, J. Appl. Phys., 1991, 69, 2849.

13. Y. Sutou, T. Kamada, Y. Saito, M. Sumiya, and J. Koike, $J$. Mater. Res. Soc. Symp. Proc., 2010, 1251, H05-8.

14. M. Dogguy, C. Carcaly, J. Rivet, and J. Flahaut, J. LessCommon Metals, 1977, 51, 181.

15. Y. Saito, Y. Sutou, and J. Koike, Appl. Phys. Lett., 2013 102, 051910.

16. T. Kamada, Y. Sutou, M. Sumiya, Y. Saito, and J. Koike, Thin Solid Films, 2012, 520, 4389.

17. G. E. Delgado, A. J. Mora, M. Pirela, A. VelásquezVelásquez, M. Villarreal, and B. J. Fernández, Phys. Stat. Sol. A, 2004, 201, 2900.

18. P. Jóvári, Y. Sutou, I. Kaban, Y. Saito, and J. Koike, Scr. Mater, 2013, 68, 122.

19. K. Kamimura, K. Kimura, S. Hosokawa, N. Happo, H. Ikemoto, Y. Sutou, S. Shindo, Y. Saito, and J. Koike, Z. Phys. Chem., 2016, 230, 433.

20. K. Kamimura, S. Hosokawa, N. Happo, H. Ikemoto, Y. Sutou, S. Shindo, Y. Saito, and J. Koike, J. Optoelectron. Adv. Mater., 2016, 18, 248.

21. J. M. Skelton, K. Kobayashi, Y. Sutou, and S. R. Elliott, Appl. Phys. Lett., 2013, 102, 224105.

22. N.-K. Chen, X.-B. Li, X.-P. Wang, M.-J. Xia, S.-Y. Xie, H.Y. Wang, Z. Song, S. Zhang, and H.-B. Sun, Acta Mater, 2015, 90, 88 .
23. S. Hosokawa, W.-C. Pilgrim, A. Höhle, D. Szubrin, N Boudet, J.-F. Bérar, and K. Maruyama, J. Appl. Phys., 2012, 111, 083517.

24. J. R. Stellhorn, B. Paulus, S. Hosokawa, W.-C. Pilgrim, N. Boudet, N. Blanc, and Y. Sutou, arXiv:1908.07297 [condmat.mtrl-sci].

25. Y. Saito, Y. Sutou, and J. Koike, Appl. Phys. Lett., 2013, 102, 051910 .

26. Y. Saito, Y. Sutou, and J. Koike, J. Phys. Chem. C, 2014, $118,26973$.

27. S. Hosokawa, Y. Wang, J.-F. Bérar, J. Greif, W.-C. Pilgrim, and K. Murase, Z. Phys. Chem., 2002, 216, 1219.

28. S. Hosokawa, J. R. Stellhorn, W.-C. Pilgrim, and J.-F. Bérar, Z. Phys. Chem., 2016, 230, 313.

29. Y. Waseda, "Novel Application of Anomalous (Resonant) $X$-Ray Scattering for Structural Characterization of Disordered Materials", 1984, Springer, Berlin.

30. S. Sasaki, "KEK Report 1989", 1989, Nat. Lab. High Energy Phys., Tsukuba, 1.

31. C. H. MacGillavry and G. D. Rieck (ed.), "International Tables for Crystallography", 2nd ed., 1968, Vol. III, Kynoch, Birmingham.

32. S. Hosokawa, I. Oh, M. Sakurai, W.-C. Pilgrim, N. Boudet, J.-F. Bérar, and S. Kohara, Phys. Rev. B, 2011, 84, 014201.

33. R. L. McGreevy and L. Pusztai, Mol. Simul., 1988, 1, 359.

34. N. Metropolis, A. W. Rosenbluth, M. N. Rosenbluth, A. H. Teller, and E. Teller, J. Phys. Chem., 1953, 21, 1087.

35. P. Pyykkö, Phys. Rev. B, 2012, 85, 024115.

36. O. Gereben, P. Jóvári, L. Temleitner, and L. Pusztai, $J$. Optoelectron. Adv. Mater, 2007, 9, 3021.

37. O. Gereben and L. Pusztai, J. Comput. Chem., 2012, 33 , 2285.

38. Y. Hiraoka, T. Nakamura, A. Hirata, E. G. Escolar, K. Matsue, and Y. Nishiura, Proc. Natl. Acad. Sci. U. S. A., 2016, 113, 7035 .

39. Y. Onodera, S. Kohara, S. Tahara, A. Masuno, H. Inoue, M. Shiga, A. Hirata, K. Tsuchiya, Y. Hiraoka, I. Obayashi, K. Ohara, A. Mizuno, and O. Sakata, J. Ceram. Soc. Jpn., 2019, 127, 853.

40. I. Petri, P. S. Salmon, and H. E. Fischer, Phys. Rev. Lett., 2000, 84, 2413.

41. J. Akola, R. O. Jones, S. Kohara, S. Kimura, K. Kobayashi, M. Takata, T. Matsunaga, R. Kojima, and N. Yamada, Phys. Rev. B, 2009, 80, 020201 .

42. S. Caravati, M. Bernasconi, T. D. Kühne, M. Krack, and M. Parrinello, Appl. Phys. Lett., 2007, 91, 171906.

43. B. Huang and J. Robertson, Phys. Rev. B, 2010, 81, 081204.

44. S. L. Roux and P. Jund, Comp. Mater. Sci., 2010, 49, 70.

45. A. F. Ioffe and A. R. Regel, Prog. Semicond., 1960, 4, 237.

46. Y. Waseda and K. Suzuki, Z. Phys. B, 1975, 20, 339.

47. J. Mizuki, K. Kakimoto, M. Misawa, T. Fukunaga and N. Watanabe, J. Phys.: Condens. Matter, 1993, 5, 3391.

48. M. Salamon (ed.), "Physics of Superionic Conductors", 1979, Springer-Verlag, Heidelberg.

49. S. Kohara, H. Tajiri, C. H. Song, K. Ohara, L. Temleitner, K. Sugimito, A. Fujiwara, L. Pusztai, T. Usuki, S. Hosokawa, Y. Benino, N. Kitamura, and K Fukumi, $J$. Phys.: Conf. Ser., 2014, 502, 012014. 